\title{
Development and validation of a broad scheme for prediction of HLA class II restricted T cell epitopes.
}

\section{Running title:}

Prediction of HLA class II restricted epitopes

\section{Authors:}

Sinu Paul ${ }^{1}$, Cecilia S. Lindestam Arlehamn ${ }^{1}$, Thomas J. Scriba ${ }^{2}$, Myles B.C. Dillon $^{1}$, Carla Oseroff ${ }^{1}$, Denise Hinz ${ }^{1}$, Denise M. McKinney ${ }^{1}$, Sebastian Carrasco Pro $^{3}$, John Sidney ${ }^{1}$, Bjoern Peters ${ }^{1}$ and Alessandro Sette ${ }^{1}$

\section{Affiliation:}

1. La Jolla Institute for Allergy and Immunology, 9420 Athena Circle, La Jolla, CA, 92037, USA

2. South African Tuberculosis Vaccine Initiative (SATVI) and School of Child and Adolescent Health, Institute of Infectious Disease and Molecular Medicine, University of Cape Town, Cape Town, South Africa

3. Laboratorio de Bioinformática y Biología Molecular, Facultad de Ciencias y Filosofía, Universidad Peruana Cayetano Heredia, Lima, Peru 


\section{Corresponding author:}

Sinu Paul

Division of Vaccine Discovery

La Jolla Institute for Allergy and Immunology

La Jolla, CA 92037 USA

Phone: (858) 752-6925

Fax: (858) 752-6987

Email: spaul@liai.org 


\section{Abstract}

Computational prediction of HLA class II restricted T cell epitopes has great significance in many immunological studies including vaccine discovery. In recent years, prediction of HLA class II binding has improved significantly but a strategy to globally predict the most dominant epitopes has not been rigorously defined. Using human immunogenicity data associated with sets of 15-mer peptides overlapping by 10 residues spanning over 30 different allergens and bacterial antigens, and HLA class II binding prediction tools from the Immune Epitope Database and Analysis Resource (IEDB), we optimized a strategy to predict the top epitopes recognized by human populations. The most effective strategy was to select peptides based on predicted median binding percentiles for a set of seven DRB1 and DRB3/4/5 alleles. These results were validated with predictions on a blind set of 15 new allergens and bacterial antigens. We found that the top $21 \%$ predicted peptides (based on the predicted binding to seven DRB1 and DRB3/4/5 alleles) were required to capture $50 \%$ of the immune response. This corresponded to an IEDB consensus percentile rank of 20.0, which could be used as a universal prediction threshold. Utilizing actual binding data (as opposed to predicted binding data) did not appreciably change the efficacy of global predictions, suggesting that the imperfect predictive capacity is not due to poor algorithm performance, but intrinsic limitations of HLA class II epitope prediction schema based on HLA binding in genetically diverse human populations. 


\section{Keywords}

Epitope prediction, HLA class II restricted T cell epitopes

\section{Abbreviations}

IEDB, Immune Epitope Database and Analysis Resource; MTB, Mycobacterium tuberculosis; HDM, House dust mite; TG, Timothy grass; TB-SD, Tuberculosis San Diego cohort; TB-CT, Tuberculosis - Cape Town cohort; LTBI, Latent Tuberculosis Infection 


\section{Introduction}

The prediction and identification of HLA class II restricted T cell epitopes is a task of significance for several different applications. These include, to name a few, efforts to elucidate the epitopes responsible for the induction of allergen specific $T$ cells, the study of immune response against complex pathogens with large genomes, such as Mycobacterium tuberculosis (MTB), or the identification and removal of unwanted epitopes in protein-based drugs.

Class II molecules are alpha/beta heterodimers encoded by four different loci in humans, DRA/DRB1, DRA/DRB3/4/5, DPA/DPB and DQA/DQB. With the exception of DRA, all other chains are highly polymorphic (Robinson et al., 2003). The extensive polymorphism of HLA class II molecules in the general population does represent a formidable obstacle to epitope identification approaches. However, it has been recognized that the majority of molecules expressed in the general population can be reconciled to a manageable number by focusing on those most frequently expressed (McKinney et al., 2013). At the same time, extensive similarities exist within the peptides bound by different allelic variants, and even across different loci (Greenbaum et al., 2011). Finally and perhaps most significantly, it has been shown that peptides capable of binding multiple HLA class II molecules (i.e. promiscuous peptides) often account for a large fraction, if not the majority, of antigen specific $T$ cell responses (Oseroff et al., 2010; Paul et al., 2013a).

Bioinformatic predictions of MHC binding capacity have proven to be a key component of various epitope identification approaches. While historically less 
impressive than the case for HLA class I, the performance of various methods for the prediction of HLA class II binding peptides has been subject to significant improvement over the last few years as more novel and sophisticated computational approaches have been implemented, as reviewed and evaluated in several studies (Paul et al., 2013a; Nielsen et al., 2010; Wang et al., 2010). However, to date, definition of an optimal strategy to employ these algorithms to allow efficient prediction of promiscuous class II restricted $\mathrm{T}$ cell epitopes, or dominant epitopes frequently recognized in an outbred cohort, has been difficult.

During the last few years we have generated $\mathrm{T}$ cell recognition data in humans for several panels of overlapping peptides completely spanning entire antigens of immunological interest. These antigens included four house dust mite allergens (referred as HDM data set) (Hinz, D., in preparation), ten allergens linked to pollen grass allergies (TG) (Oseroff et al., 2010), four MTB antigens recognized by healthy donors with latent MTB infection (LTBI) from the San Diego region (TB-SD) (Arlehamn et al., 2012), and eleven different MTB antigens recognized by healthy donors with LTBI from the Cape Town (South Africa) region (TB-CT) (Mc Kinney, D., in preparation). Each set of peptides was tested with similar methodology, in 20-40 different HLA typed individuals of diverse ethnicity. Overall, a total of 1151 peptides were tested, in studies involving more than 95 donors.

In the present study we have utilized these data sets to perform an evaluation of different strategies to implement HLA binding predictions for the purpose of selecting epitopes with the capacity to elicit HLA class II restricted T 
cell immune responses. To validate the approach defined herein, independent blind analyses were subsequently performed using overlapping peptide sets spanning six different cockroach allergens (Oseroff et al., 2012; Dillon, M., in preparation) and the five antigens included in the vaccine against whooping cough (Bordetella pertussis) (Dillon, M., in preparation). 


\section{Materials and methods}

\subsection{Immunogenicity studies}

Sets of overlapping 15 or 16 mer peptides spanning various allergen and bacterial antigens were screened for immune reactivity as previously described (Oseroff et al., 2010; Arlehamn et al., 2012; Oseroff et al., 2012). Antigen specific cytokine production in donor peripheral-blood mononuclear cells (PBMC) was measured in dual or single ELISPOT assays. Responses to timothy grass, cockroach and house dust mite peptides were measured following in vitro stimulation with respective allergen extracts, and responses to Bordetella pertussis peptides following stimulation with corresponding vaccine antigens. Responses to mycobacterial antigens were analyzed ex vivo. Peptide specific responses were expressed as spot-forming cells (SFCs)/10 6 PBMC. Donors were HLA typed at each class II locus to four-digit resolution by SSO/SSP HLA typing (One Lambda reagents, Canoga Park, CA, USA) or deep sequencing methods (2012McKinney et al., submitted).

\subsection{MHC purification and binding Assays}

The binding affinity of peptides in TG and TB-SD data sets to the 26 most frequent alleles was experimentally determined. Quantitative measurement of peptide binding capacity for HLA class II molecules was performed in competition assays based on the inhibition of binding of a high affinity radiolabeled peptide to purified MHC molecules. Purification of class II MHC molecules by affinity 
chromatography, and the performance of binding assays were done essentially as detailed elsewhere (Sidney et al., 2013). Briefly, EBV transformed homozygous cell lines were used as sources of MHC molecules. A high affinity radiolabeled peptide $(0.1-1 \mathrm{nM})$ was co-incubated at room temperature or $37^{\circ} \mathrm{C}$ with purified MHC in the presence of a cocktail of protease inhibitors. Following a two-day incubation, MHC bound radioactivity was determined by capturing MHC/peptide complexes on Ab coated Lumitrac 600 plates (Greiner Bio-one, Frickenhausen, Germany), and measuring bound cpm using the TopCount (Packard Instrument Co., Meriden, CT) microscintillation counter. The concentration of peptide yielding $50 \%$ inhibition of the binding of the radiolabeled peptide was calculated. Under the conditions utilized, where $[$ label $]<[\mathrm{MHC}]$ and IC50 $\geq[\mathrm{MHC}]$, the measured IC50 values are reasonable approximations of the true Kd values. Each competitor peptide was tested at six different concentrations covering a 100,000-fold range, and in three or more independent experiments. As a positive control, the unlabeled version of the radiolabeled probe was also tested in each experiment.

\subsection{Prediction of binding affinity}

Peptide binding affinity for HLA class II alleles was predicted using the MHC II binding prediction tool available at the IEDB (www.iedb.org) (Vita et al., 2014, Zhang et al., 2008, Kim et al., 2012). Allele-specific consensus percentile ranks of all algorithms queried by the IEDB tool were utilized (Wang et al., 2010). A percentile rank is generated by comparing the selected peptide's predicted 
binding affinity against that of a large set of similarly sized peptides randomly selected from the SWISS-PROT database (Kim et al., 2012). Percentile rank provides a uniform scale allowing comparisons across different predictors. A lower percentile rank value indicates higher affinity. In the case of consensus method, median of the percentile ranks of the three methods involved is considered as the IEDB consensus percentile rank.

\subsection{Correction for epitope redundancy}

Responses against two consecutive peptides are often due to the same minimal epitope. To avoid counting the same epitope twice, two consecutive responses with magnitudes within 2.5 -fold of each other were merged into a single antigenic region, and the higher SFC value utilized. The region was considered successfully predicted if either of the two peptides was predicted, and "credit" for prediction was given only once. 


\section{Results and discussion}

\subsection{Evaluation of optimal prediction strategies for HLA class II epitopes}

Predicted binding affinity of peptides in the data sets (Table 1) for a previously described set of $26 \mathrm{HLA}$ class II alleles that are most frequent in the general worldwide population (Greenbaum et al., 2011) (Table 2), was determined as described above. To evaluate the efficacy of various approaches to employing these predictions to identify the most dominant epitope responses, the percentage (fraction) of peptides in each data set needed to capture $50 \%$ of the total response $(50 \%$ of the total SFC values in the data set - expressed as SFCs $/ 10^{6}$ PBMCs) was utilized as a performance metric.

Table 1: Data sets used in the analyses

\begin{tabular}{|l|l|l|l|l|l|l|l|}
\hline Data set & $\begin{array}{l}\text { No. } \\
\text { of } \\
\text { antig } \\
\text { ens }\end{array}$ & Antigens & $\begin{array}{l}\text { No. of } \\
\text { peptide } \\
\text { s per } \\
\text { antigen }\end{array}$ & $\begin{array}{l}\text { No. of } \\
\text { peptid } \\
\text { es per } \\
\text { data } \\
\text { set }\end{array}$ & $\begin{array}{l}\text { No. of } \\
\text { donors }\end{array}$ & $\begin{array}{l}\text { Ethni } \\
\text { city }\end{array}$ & Reference \\
\hline HDM & 4 & $\begin{array}{l}\text { proDer } p \\
1.0105 \\
\text { proDer } f \\
1.0101 \\
\text { Der } p \\
2.0101 \\
\text { Der } f\end{array}$ & 59 & $156^{\star}$ & 20 & White & $\begin{array}{l}\text { Hinz, D., in } \\
\text { preparation }\end{array}$ \\
\hline T.0103 & 24 & 24 & & & \\
\hline TG & 10 & $\begin{array}{l}\text { Phl p 1 } \\
\text { Phl p 2 } \\
\text { Phl p 3 } \\
\text { Phl p 4 }\end{array}$ & $\begin{array}{l}51 \\
23\end{array}$ & 425 & 25 & $\begin{array}{l}\text { Mixed } \\
\text { (pred } \\
\text { omina } \\
\text { ntly }\end{array}$ & $\begin{array}{l}\text { Oseroff et } \\
\text { al., 2010 }\end{array}$ \\
\hline
\end{tabular}




\begin{tabular}{|c|c|c|c|c|c|c|c|}
\hline & & $\begin{array}{l}\text { Phl p } \\
5.0103 \\
\text { Phl p } 6 \\
\text { Phl p } 7 \\
\text { Phl p } 11 \\
\text { Phl p } 12 \\
\text { Phl p } 13\end{array}$ & $\begin{array}{l}61 \\
26 \\
14 \\
27 \\
25 \\
77\end{array}$ & & & white) & \\
\hline TB-SD & 4 & $\begin{array}{l}\text { Rv1038c } \\
\text { Rv2031c } \\
\text { Rv3874 } \\
\text { Rv3875 }\end{array}$ & $\begin{array}{l}9 \\
27 \\
18 \\
17\end{array}$ & 71 & 18 & Mixed & $\begin{array}{l}\text { Arlehamn } \\
\text { et al., } 2012\end{array}$ \\
\hline TB-CT & 11 & $\begin{array}{l}\text { Rv0125 } \\
R v 0288 \\
R v 1196 \\
R v 1813 c \\
R v 1886 c \\
R v 2608 \\
R v 2660 c \\
R v 3619 \\
\text { Rv3620c } \\
\text { Rv3804c } \\
\text { Rv3875 }\end{array}$ & $\begin{array}{l}69 \\
18 \\
77 \\
27 \\
63 \\
114 \\
13 \\
17 \\
18 \\
66 \\
17\end{array}$ & 499 & 32 & & $\begin{array}{l}\text { McKinney, } \\
\text { D. M., in } \\
\text { preparation }\end{array}$ \\
\hline Cockroach & 6 & $\begin{array}{l}\text { Bla g } 1 \\
\text { Bla g } 2 \\
\text { Bla g } 4 \\
\text { Bla g } 5 \\
\text { Bla g } 6 \\
\text { Bla g } 7\end{array}$ & $\begin{array}{l}189 \\
69 \\
35 \\
39 \\
76 \\
55\end{array}$ & 463 & 19 & & $\begin{array}{l}\text { Oseroff et } \\
\text { al., } 2012\end{array}$ \\
\hline Pertussis & 9 & $\begin{array}{l}\text { fhaB } \\
\text { fim2 } \\
\text { fim3 } \\
\text { prn } \\
\text { ptxA } \\
\text { ptxB } \\
\text { ptxC } \\
\text { ptxD } \\
\text { ptxE }\end{array}$ & $\begin{array}{l}468 \\
26 \\
25 \\
131 \\
40 \\
30 \\
28 \\
21 \\
16\end{array}$ & 785 & 23 & & $\begin{array}{l}\text { Dillon, } \\
\text { M.B.C., in } \\
\text { preparation }\end{array}$ \\
\hline
\end{tabular}


*10 peptides are shared by different antigens in the Der p/f data set. Thus, the total no. of unique peptides is 10 less than the sum of peptides in individual antigens.

Table 2: 26 HLA class II alleles that are most frequent in the general worldwide population, and thus were included in the analyses

\begin{tabular}{|c|c|c|c|}
\hline Locus & Allele & $\begin{array}{l}\text { Phenotype } \\
\text { frequency }\end{array}$ & $\begin{array}{l}\text { Gene } \\
\text { frequency }\end{array}$ \\
\hline \multirow[t]{12}{*}{ DRB1 } & DRB ${ }^{*} 0101$ & 5.44 & 2.76 \\
\hline & DRB1*0301 & 13.72 & 7.11 \\
\hline & DRB1*0401 & 4.58 & 2.32 \\
\hline & DRB1 ${ }^{\star} 0405$ & 6.15 & 3.13 \\
\hline & DRB1*0701 & 13.52 & 7.01 \\
\hline & DRB1*0802 & 4.87 & 2.46 \\
\hline & DRB1*0901 & 6.17 & 3.13 \\
\hline & DRB1*1101 & 11.84 & 6.11 \\
\hline & DRB1*1201 & 3.94 & 1.99 \\
\hline & DRB1*1302 & 7.71 & 3.93 \\
\hline & DRB1*1501 & 12.18 & 6.29 \\
\hline & Total & 71.09 & 46.23 \\
\hline \multirow[t]{5}{*}{ DRB3/4/5 } & DRB3 $^{*} 0101$ & 26.12 & 14.04 \\
\hline & DRB3*0202 & 34.25 & 18.92 \\
\hline & DRB4*0101 & 41.75 & 23.68 \\
\hline & DRB5*0101 & 15.98 & 8.34 \\
\hline & Total & 87.73 & 77.30 \\
\hline \multirow[t]{7}{*}{ DQA1/DQB1 } & DQA1*0501/DQB1*0201 & 11.29 & 5.81 \\
\hline & DQA1*0501/DQB1*0301 & 35.14 & 19.47 \\
\hline & $\mathrm{DQA1}{ }^{*} 0301 / \mathrm{DQB} 1{ }^{*} 0302$ & 19.05 & 10.03 \\
\hline & DQA1*0401/DQB1*0402 & 12.78 & 6.61 \\
\hline & DQA1*0101/DQB1*0501 & 14.65 & 7.61 \\
\hline & DQA $1{ }^{*} 0102 / \mathrm{DQB} 1{ }^{*} 0602$ & 14.59 & 7.58 \\
\hline & Total & 81.61 & 57.11 \\
\hline \multirow[t]{2}{*}{ DPB1 } & DPA $1 * 02: 01 / D P B 1{ }^{*} 01: 01$ & 16.01 & 8.35 \\
\hline & DPA1*01:03/DPB1*02:01 & 17.47 & 9.15 \\
\hline
\end{tabular}




\begin{tabular}{|c|c|c|}
\hline $\begin{array}{l}\text { DPA } 1{ }^{*} 01 / \mathrm{DPB} 1{ }^{*} 04: 01 \\
\text { DPA } 1{ }^{*} 03: 01 / \mathrm{DPB} 1{ }^{*} 04: 02 \\
\text { DPA } 1{ }^{*} 02: 01 / \mathrm{DPB} 1{ }^{*} 05: 01\end{array}$ & $\begin{array}{l}36.20 \\
41.63 \\
21.68 \\
\end{array}$ & $\begin{array}{l}20.13 \\
23.60 \\
11.50\end{array}$ \\
\hline Total & 94.49 & 76.53 \\
\hline
\end{tabular}

As a first approach, we considered the "promiscuous binding capacity" of each peptide, where promiscuity is defined by the number of alleles bound (i.e., peptides binding more alleles being more promiscuous binders). For this purpose, a peptide was considered binder for a specific allele if its IEDB predicted consensus percentile rank was $\leq 20$. This approach was originally devised by us based on a single dataset (TG, Oseroff et al., 2010). Using this approach, as shown in Figures $\mathbf{1}$ a and b, an average of $30.91 \%$ (range 25.35\%$40.08 \%$ ) peptides were needed to capture $50 \%$ of the total response in the data set.

As a second approach, we considered the "median consensus percentile rank" of each peptide, defined as the median of the IEDB consensus percentile ranks predicted for the set of 26 selected alleles. This approach was the most effective, with the top $26.26 \%$ (range $16.90 \%-38.38 \%$ ) of the peptides capturing $50 \%$ of the total response (Figure 1a, c). 
Figure 1a: Performance of two approaches for implementing HLA class II binding predictions to identify $\mathbf{T}$ cell epitopes. The $\%$ of peptides needed for each method to identify a panel of epitopes accounting for $50 \%$ of the total antigen specific response (SFC) is shown for 4 different systems, as described in the text: HDM (House dust mite), TG (Timothy grass), TB-SD (MTB), and TB-CT (MTB). Blue bars show performance based on ranking peptides according to the median consensus percentile rank against a panel of the 26 most common HLA class II alleles. Red bars show performance based on ranking peptides according to the number of alleles predicted to bind (promiscuity). A lower $\%$ of peptides indicates better performance.

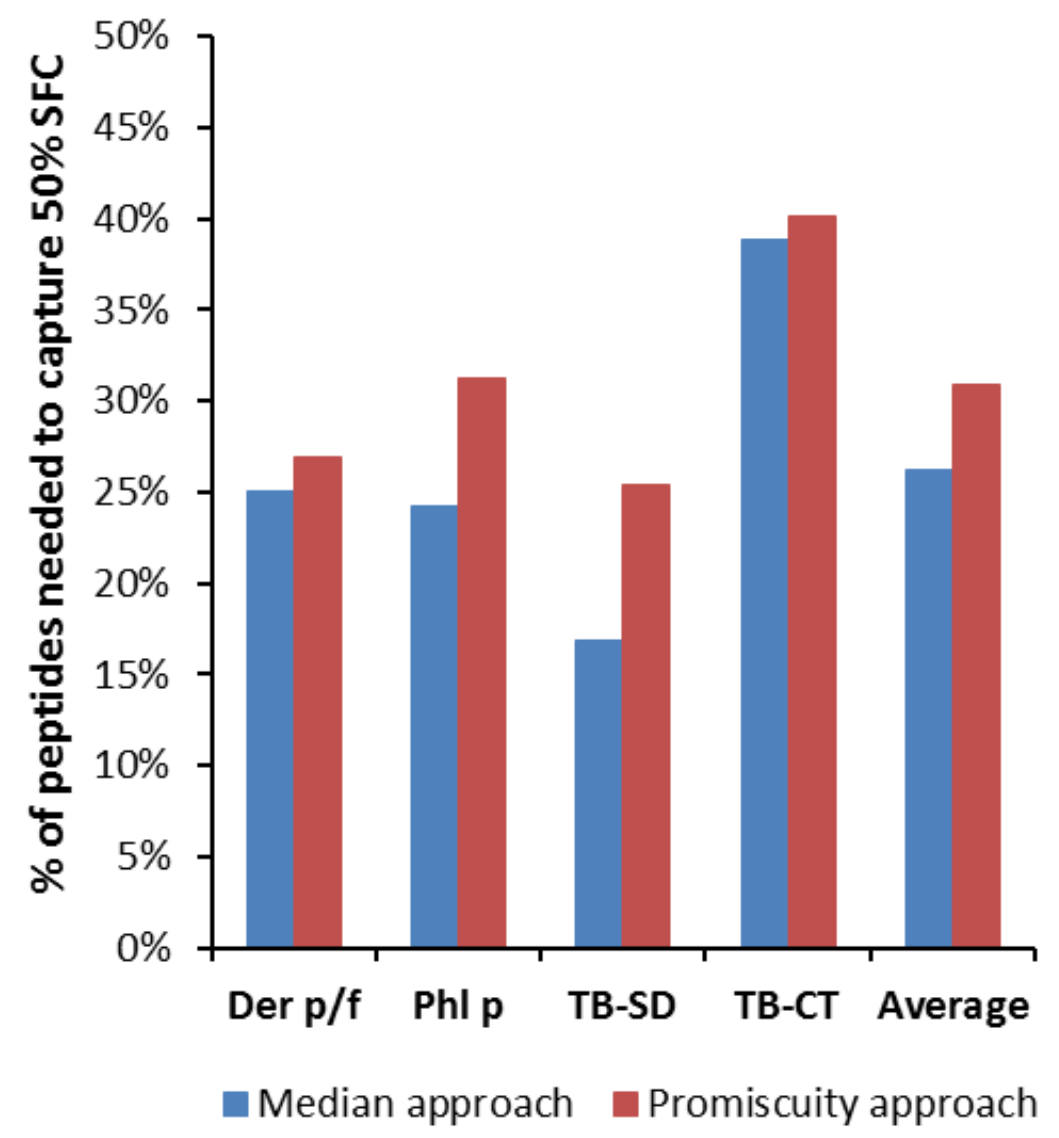


Figure $1 \mathrm{~b}:$ The $\%$ of response predicted as a function of the $\%$ of the total peptides predicted for the four data sets with the "promiscuous binding capacity" approach using the 26 most frequent class II alleles.

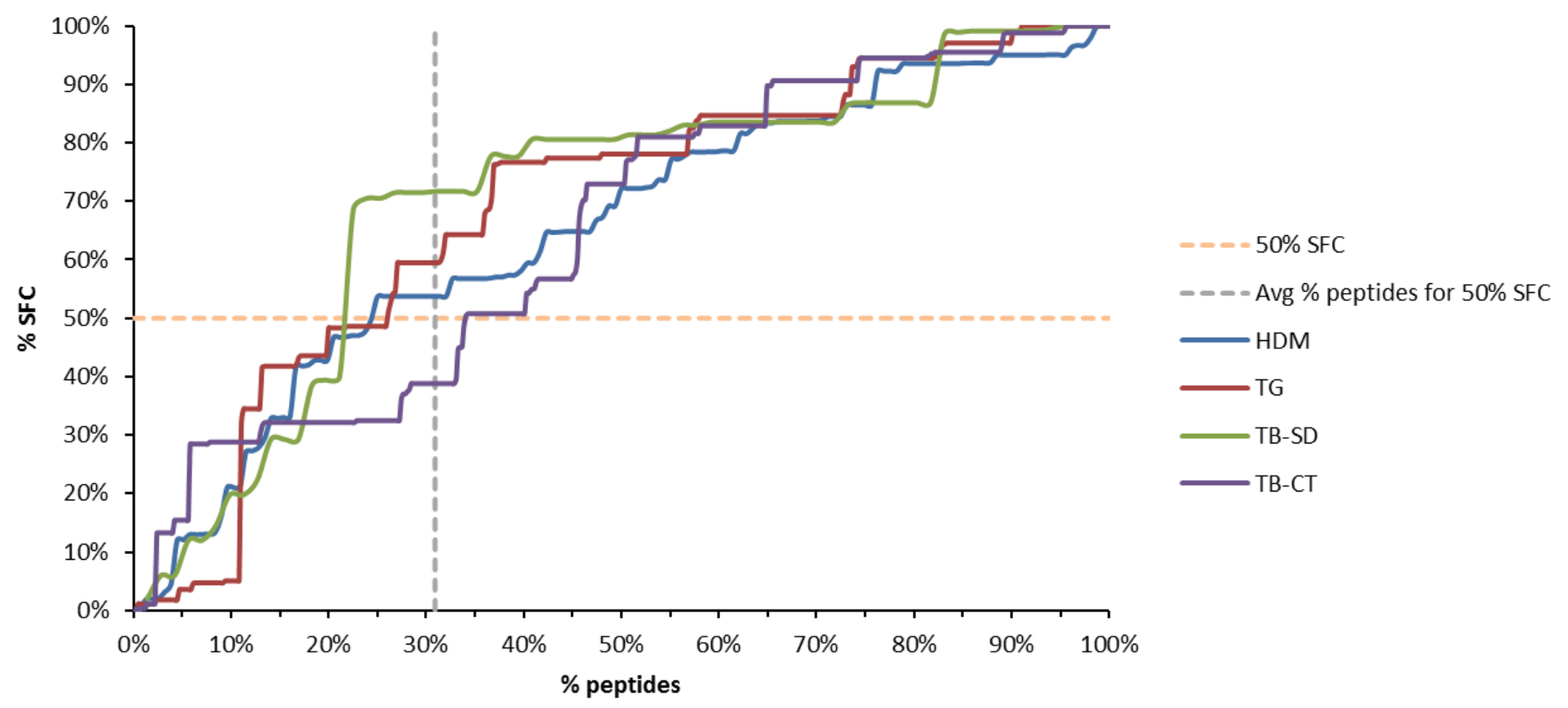


Figure 1c: The $\%$ of response predicted as a function of the $\%$ of the total peptides predicted for the four data sets with the "Median percentile" approach using the 26 most frequent class II alleles.

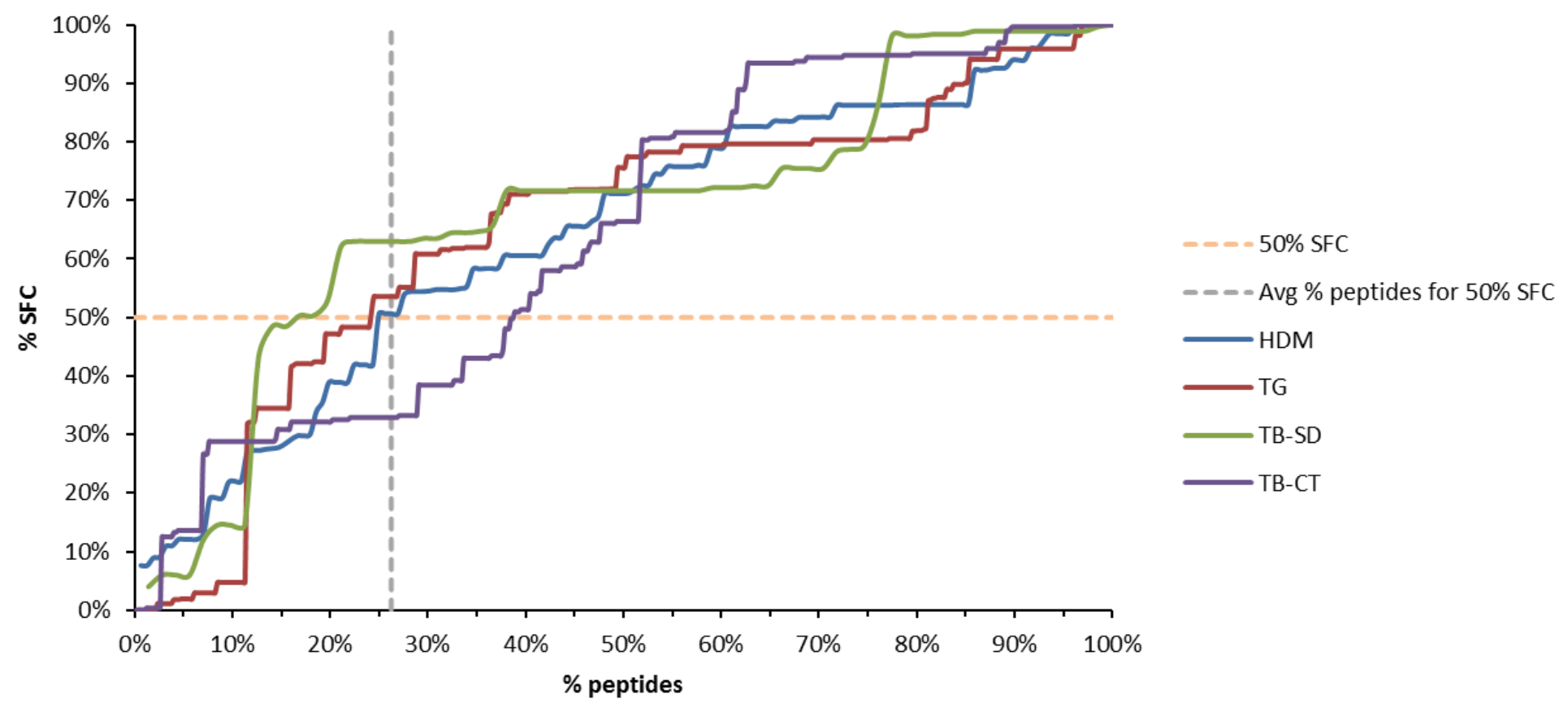

3.2 Comparison of the median percentile rank approach with best percentile and allele specific binding thresholds

In addition to the promiscuous binding capacity (promiscuity) and median consensus percentile rank, other strategies were also evaluated. Considering that the dominance of a particular peptide might be a reflection of very high binding affinity for a given allele rather than promiscuity, one approach we tested was based on "best percentile rank". In this approach, the peptides were sorted based on the best percentile rank (the lowest percentile rank value among the 26 most frequent alleles) and the percentage of peptides required to capture $50 \%$ of 
total SFC was identified. This method required on average $27.05 \%$ of the top peptides to capture $50 \%$ of the response.

In yet another approach, we followed a strategy based on allele-specific binding affinity thresholds that had improved the efficacy of class I predictions (Paul et al., 2013b). For this analysis, all previously identified 15-mer epitopes with defined HLA class II restriction were retrieved from the IEDB. The allelespecific thresholds were estimated for each of the 26 alleles in terms of binding affinity predicted by SMM_align method $\left(\mathrm{IC}_{50}\right)$ (Nielsen et al., 2007), taking into account the number of predicted binders in the set of epitopes retrieved from the IEDB (based on a general threshold of $\mathrm{IC}_{50}$ 1000nM) and the SMM_align IC $\mathrm{C}_{50}$ value demarcating the top $75 \%$ peptides of the same epitope set. The total promiscuity for each peptide was then recalculated based on the allele-specific thresholds and the fraction of peptides required to capture $50 \%$ response was identified. This approach required $33.58 \%$ of peptides to capture $50 \%$ response.

Both these approaches were found to be less efficient than the median consensus percentile rank method, requiring on average $27.05 \%$ and $33.58 \%$ peptides respectively (vs. 26.26\%), to capture 50\% response based on the 26 most frequent alleles (data not shown).

\subsection{Exclusion of DP locus improves predictive efficacy}

As different HLA class II loci appear to contribute differentially to human responses (Oseroff et al., 2010), we hypothesized that examining the performance as a function of the class II locus may improve predictions. The 
average $\%$ of peptides required to capture $50 \%$ SFC for different combinations of DRB1, DRB3/4/5, DQ, and DP alleles are shown in Figure 2. The best results $(23.82 \%)$ were obtained when DP alleles were left out. The lower performance of methods incorporating DP molecules might be due to the fact that less binding data is available for these molecules leading to inferior prediction algorithms or it could be that DP molecules are less often restricting elements for dominant $\mathrm{T}$ cell responses.

Figure 2: Performance of the "median consensus percentile rank" approach as a function of variable inclusion of the DP, DQ, DRB1 and DRB3/4/5 loci. The performance was best when DP locus was excluded (red bar).

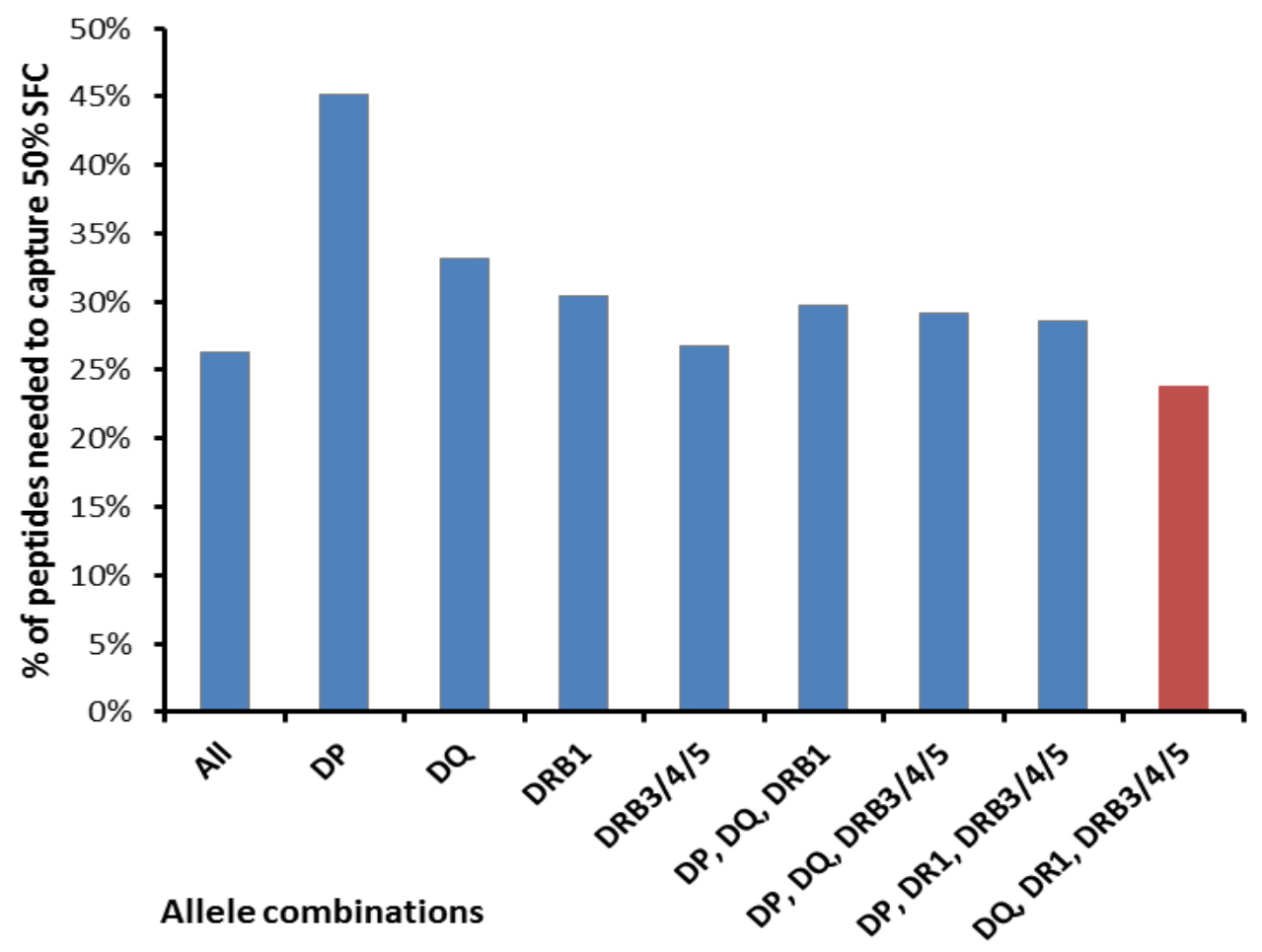




\subsection{Optimal results obtained with a set of seven DRB1 and DRB3/4/5 alleles}

We next examined the effect of varying the specific alleles included in the prediction panel. Frequency thresholds for inclusion were varied independently for each locus (i.e., DQ, DRB1 and DRB3/4/5). The best results $(21.41 \%$ of peptides needed to capture $50 \%$ SFC) were observed when the three DRB1 alleles with frequency $\geq 12 \%\left(\mathrm{DRB} 1{ }^{*} 03: 01, \mathrm{DRB} 1{ }^{*} 07: 01, \mathrm{DRB} 1{ }^{*} 15: 01\right)$ were used along with the four DRB3/4/5 alleles (DRB3*01:01, DRB3*02:02, DRB4 $^{*} 01: 01$, DRB5 $^{\star} 01: 01$ ) (data not shown). This empirical optimization is probably reflective of the fact that $\mathrm{DR}$ alleles are the most dominant locus restricting HLA class II responses in humans. It is noteworthy that the seven allelic variants cover the main HLA class II supertypes (Greenbaum et al., 2011).

\subsection{Predictions based on alleles frequent in specific donor cohorts}

HLA frequencies vary in cohorts of individuals with different ethnicity. Accordingly, we examined the performance of the "median consensus percentile rank" predictions utilizing alleles custom selected for the cohorts in which the peptides were tested. Specifically, we included all alleles with frequencies of $\geq$ $10 \%$ in a given donor population. As above, the best results (19.69\%) were obtained using only DRB1 and DRB3/4/5 alleles (Figure 3). At the same time, the improvement seen when using cohort specific allele sets is minor suggesting that tailoring the prediction to a specific population has limited value. 
Figure 3: Average $\%$ of peptides required to capture $50 \%$ SFC for different allele combinations using the alleles with frequency $>10 \%$ in each specific corresponding donor cohort. The prediction was best when alleles from DRB1 and DRB3/4/5 alleles were used (red bar)

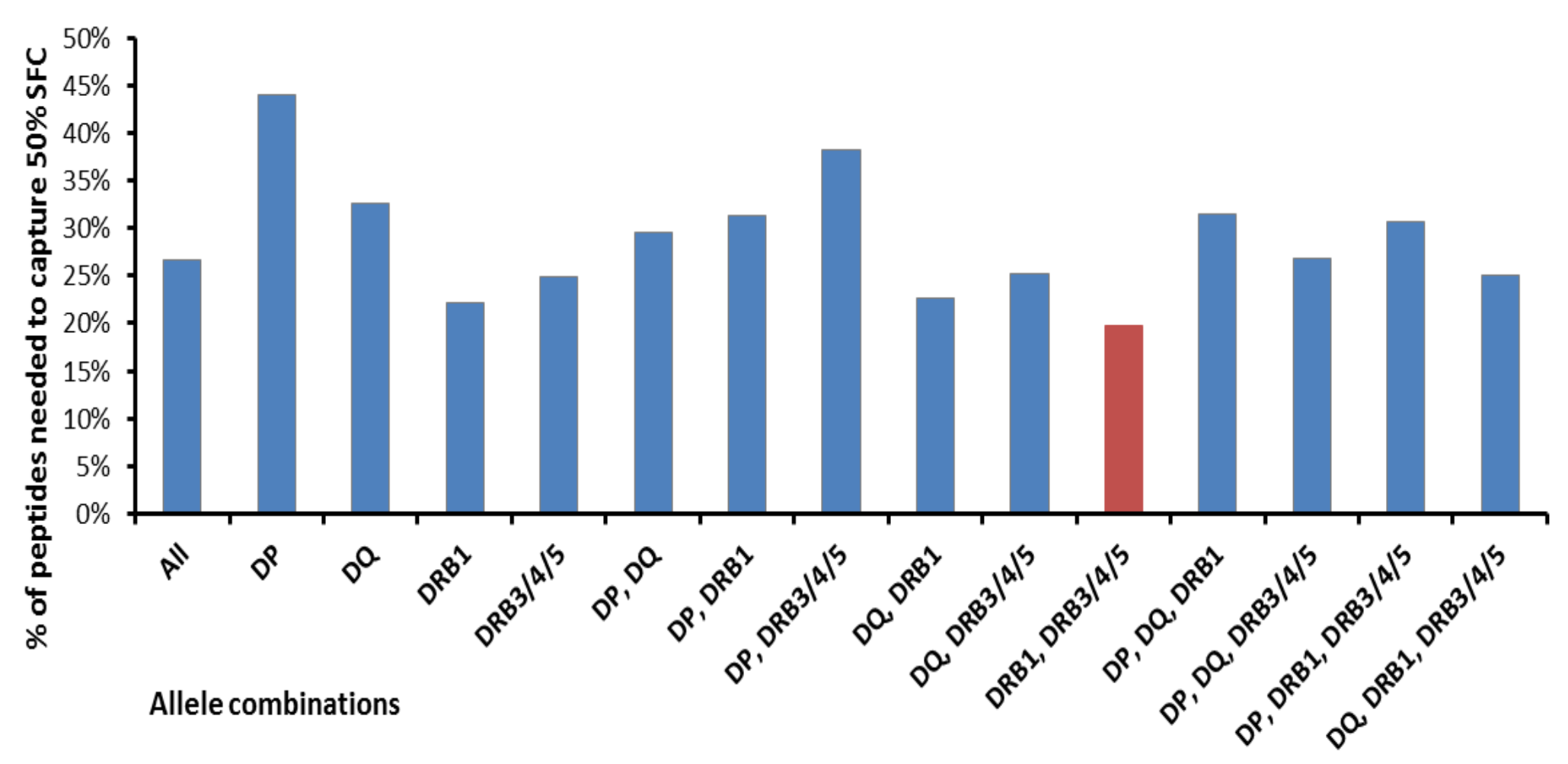




\subsection{Defining a universal prediction threshold}

The percent of total peptides required to capture $50 \%$ of the response, as calculated here on a protein-by-protein basis, is not available when considering individual peptides. To derive a standard prediction threshold, we calculated the median IEDB consensus percentile rank, using predictions for the seven DRB1 and DRB3/4/5 alleles highlighted above, associated with the selected set of peptides yielding $50 \%$ of the response. This value was found to be 20.0 (median consensus percentile rank from the seven selected alleles).

\subsection{Validation of the results with blind prediction using new data sets}

The analyses above suggested that the optimal approach for efficient selection of epitope candidates would be based on determining the median consensus percentile rank across a selected panel of seven DR alleles (3 DRB1 alleles with frequency $\geq 12 \%$ in conjunction with 4 DRB3/4/5 alleles). To validate these results we examined overlapping peptides for two additional sets of proteins of immunological interest: 1) cockroach allergens and 2) acellular pertussis vaccine antigens.

When the range of approaches tried above was implemented against the two blind sets, the best performance was again achieved with the "median consensus percentile rank" approach. When the universal median IEDB consensus percentile threshold defined above (20.0) with the panel of seven DR alleles was utilized, the average \% of SFC captured using peptides with median 
IEDB consensus percentile rank $\leq 20.0$ was found to be $48.55 \%$, confirming the validity of this prediction threshold.

\subsection{Comparison with experimentally measured binding data}

The analysis showed that on average, that approximately to top $\sim 20 \%$ scoring peptides are needed to capture $50 \%$ of the immune response. In order to examine whether this rather high number of peptides is due to lower efficacy of HLA class II binding prediction algorithms, we compared the performance based on predicted binding affinity with that of experimentally measured binding affinity. For this analysis predicted and measured binding affinity for the seven selected DR alleles was assessed in the context of two cohorts (TG and TB-SD).

It was found that the peptide selection strategy based on the predicted binding affinity required actually fewer peptides to capture $50 \%$ of the immune response, compared to the measured binding affinity $(15.63 \%$ vs. $32.86 \%$ peptides) (Figure 4). No significant difference was observed when binding data from two other allele categories were used (the most frequent panel alleles and the panel alleles excluding DP locus). This shows that the overall lower efficacy in prediction of HLA class II immunogenicity is an inherent issue of class II alleles rather than underperformance of HLA class II binding prediction algorithms. 
Figure 4: Comparison of the prediction performance based on experimentally measured binding data with that of predicted binding data for TG and TB-SD data sets. Blue bars show the $\%$ of peptides needed to capture $50 \%$ SFC using experimentally measured HLA class II binding data while red bars show the same using predicted binding data. The prediction strategy using 7 selected alleles performed better with predicted binding compared to measured binding.

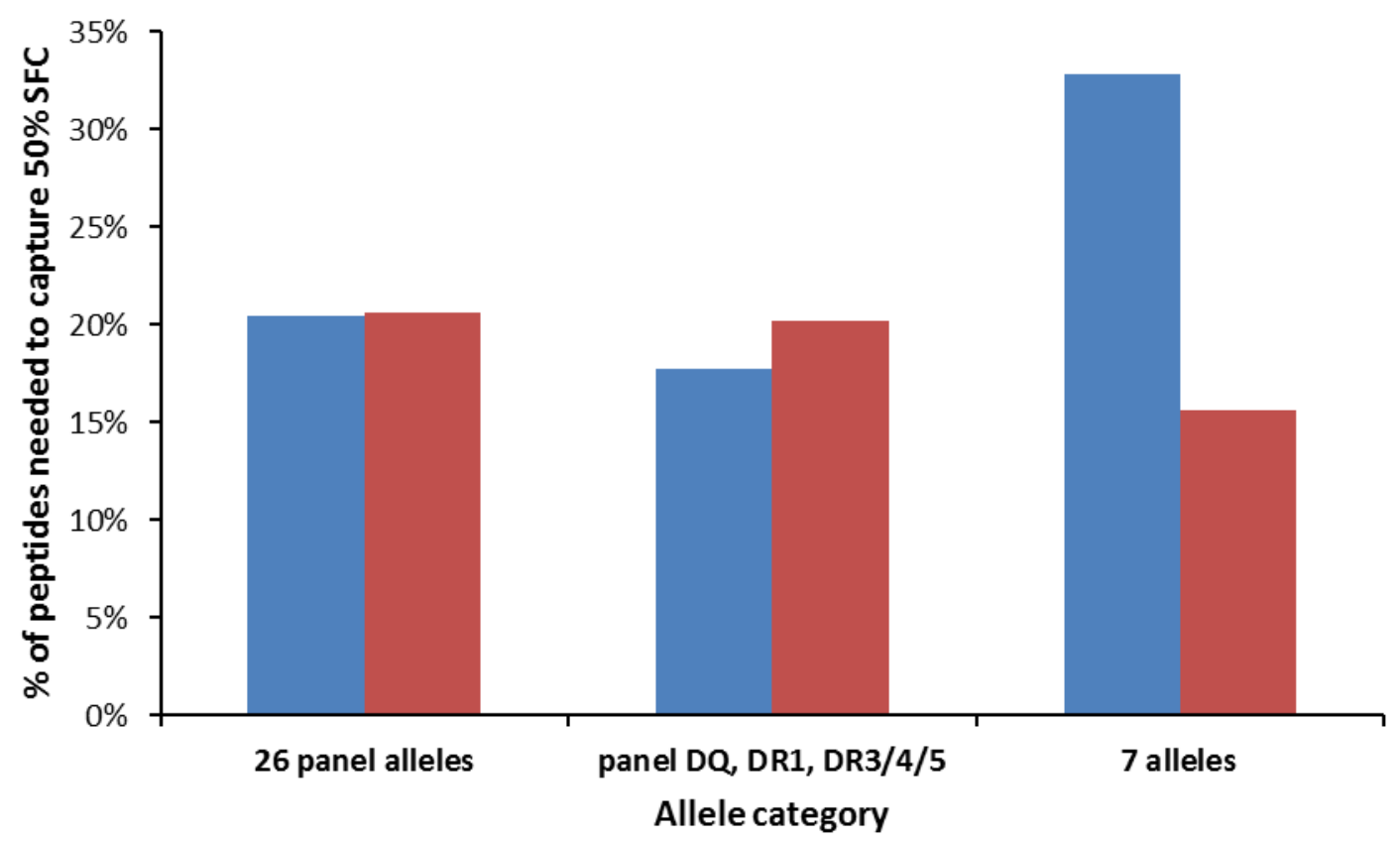




\section{Conclusions}

We scrutinized the use of HLA class II binding predictions to identify sets of epitopes with high immunological activity. The results validate previous observations that promiscuous binders account for a large fraction of the total response. However, in comparison to HLA class I predictions, the results are sobering, as the overall performance is remarkably less effective. This is in line with other recent studies (Chaves et al., 2012). We considered the possibility that these results may be due to a generally lower performance of class II binding prediction algorithms. However when actual binding data, as opposed to predicted binding data, was used, no significant improvement was noted. These results suggest that the imperfect predictive capacity is due to intrinsic limitations of HLA class II epitope prediction schema based on HLA binding in genetically diverse human populations.

At the same time, our results provide guidance for practical implementation of predictions, and identify specific subsets of HLA molecules that are most effectively considered by prediction schemes. The synthesis of approximately $20 \%$ of the peptides in a set of 15 -mer peptides overlapping by 10 residues allows covering a 200-residue protein (otherwise covered by 38 overlapping peptides) with 8 peptides, which still affords significant cost savings, and enables the screening of large genomes with experimental designs based on predicted epitope peptide pools. 


\section{Acknowledgements}

This project has been funded with federal funds from the National Institute of Allergy and Infectious Diseases, National Institutes of Health, Department of Health and Human Services under Contract Numbers HHSN272201200010C (IEDB), HHSN272200900044C (TB), HHSN272200700048C (Allergy), HHSN272200900052C (ICAC2), HHSN272201000052I (Rho) and UM1Al114271 (ICAC3) and Grant number NIH U19 Al100275 (Allergy) in addition to Global Health Grant OPP1066265 from Bill \& Melinda Gates Foundation. 


\section{References}

1. Arlehamn, C.S., Sidney, J., Henderson, R., Greenbaum, J.A., James, E.A., Moutaftsi, M., Coler, R., McKinney, D.M., Park, D., Taplitz, R., Kwok, W.W., Grey, H., Peters, B., Sette, A., 2012. Dissecting mechanisms of immunodominance to the common tuberculosis antigens ESAT-6, CFP10, Rv2031c (hspX), Rv2654c (TB7.7), and Rv1038c (EsxJ). J. Immunol. 188, $5020-5031$.

2. Chaves, F.A., Lee, A.H., Nayak, J.L., Richards, K.A., Sant, A.J., 2012. The utility and limitations of current Web-available algorithms to predict peptides recognized by CD4 T cells in response to pathogen infection. J. Immunol. 188, 4235-4248.

3. Greenbaum, J., Sidney, J., Chung, J., Brander, C., Peters, B., Sette, A., 2011. Functional classification of class II human leukocyte antigen (HLA) molecules reveals seven different supertypes and a surprising degree of repertoire sharing across supertypes. Immunogenetics 63.6, 325-335.

4. Kim, Y., Ponomarenko, J., Zhu, Z., Tamang, D., Wang, P., Greenbaum, J., Lundegaard, C., Sette, A., Lund, O., Bourne, P.E., 2012. Immune epitope database analysis resource. Nucleic Acids Res. 40, W525-W530.

5. McKinney, D.M., Southwood, S., Hinz, D., Oseroff, C., Arlehamn, C.S.L., Schulten, V., Taplitz, R., Broide, D., Hanekom, W.A., Scriba, T.J., 2013. A strategy to determine HLA class II restriction broadly covering the DR, DP, and DQ allelic variants most commonly expressed in the general population. Immunogenetics $65.5,1-14$. 
6. Nielsen, M., Lund, O., Buus, S., Lundegaard, C., 2010. MHC Class II epitope predictive algorithms. Immunology 130, 319-328.

7. Nielsen, M., Lundegaard, C., Lund, O., 2007. Prediction of MHC class II binding affinity using SMM-align, a novel stabilization matrix alignment method. BMC Bioinformatics 8, 238.

8. Oseroff, C., Sidney, J., Kotturi, M.F., Kolla, R., Alam, R., Broide, D.H., Wasserman, S.I., Weiskopf, D., McKinney, D.M., Chung, J.L., 2010. Molecular determinants of $\mathrm{T}$ cell epitope recognition to the common Timothy grass allergen. The Journal of Immunology 185, 943-955.

9. Oseroff, C., Sidney, J., Tripple, V., Grey, H., Wood, R., Broide, D.H., Greenbaum, J., Kolla, R., Peters, B., Pomés, A., 2012. Analysis of T cell responses to the major allergens from German cockroach: Epitope specificity and relationship to IgE production. The Journal of Immunology $189,679-688$.

10. Paul, S., Kolla, R.V., Sidney, J., Weiskopf, D., Fleri, W., Kim, Y., Peters, B., Sette, A., 2013a. Evaluating the Immunogenicity of Protein Drugs by Applying In Vitro MHC Binding Data and the Immune Epitope Database and Analysis Resource. Clinical and Developmental Immunology 2013.

11. Paul, S., Weiskopf, D., Angelo, M.A., Sidney, J., Peters, B., Sette, A., 2013b. HLA class I alleles are associated with peptide-binding repertoires of different size, affinity, and immunogenicity. J. Immunol. 191, 5831-5839.

12. Robinson, J., Waller, M.J., Parham, P., de Groot, N., Bontrop, R., Kennedy, L.J., Stoehr, P., Marsh, S.G., 2003. IMGT/HLA and IMGT/MHC: 
sequence databases for the study of the major histocompatibility complex. Nucleic Acids Res. 31, 311-314.

13. Sidney, J., Southwood, S., Moore, C., Oseroff, C., Pinilla, C., Grey, H.M., Sette, A., 2013. Measurement of MHC/Peptide Interactions by Gel Filtration or Monoclonal Antibody Capture. Current protocols in immunology, 18.3.1-18.3.36.

14. Vita, R., Overton, J.A., Greenbaum, J.A., Ponomarenko, J., Clark, J.D., Cantrell, J.R., Wheeler, D.K., Gabbard, J.L., Hix, D., Sette, A., Peters, B., 2014. The immune epitope database (IEDB) 3.0. Nucleic Acids Res. 2014. 15. Wang, P., Sidney, J., Kim, Y., Sette, A., Lund, O., Nielsen, M., Peters, B., 2010. Peptide binding predictions for HLA DR, DP and DQ molecules. BMC Bioinformatics 11.1, 568.

16.Zhang, Q., Wang, P., Kim, Y., Haste-Andersen, P., Beaver, J., Bourne, P.E., Bui, H.H., Buus, S., Frankild, S., Greenbaum, J., Lund, O., Lundegaard, C., Nielsen, M., Ponomarenko, J., Sette, A., Zhu, Z., Peters, B., 2008. Immune epitope database analysis resource (IEDB-AR). Nucleic Acids Res. 36, W513-8. 